\section{Lasiodiplodia theobromae keratitis: a rare fungi from eastern India}

\author{
Suman Saha, ${ }^{1}$ Jayangshu Sengupta, ${ }^{2}$ \\ Debdulal Banerjee, ${ }^{3}$ Archana Khetan ${ }^{2}$ \\ 1 Ocular Microbiology Services and \\ ${ }^{2}$ Cornea Services, Priyamvada Birla \\ Aravind Eye Hospital, Kolkata; \\ ${ }^{3}$ Department of Botany and Forestry, \\ Vidyasagar University, Midnapore, West \\ Bengal, India
}

\begin{abstract}
We report here a case of a 32-year old female with a history of minor trauma presented with total corneal ulcer and hypopyon in the left eye. Microbiological examination of corneal scraping showed refractile hyphae with asepted branching filaments and black pigmented colonies in multiple solid agar medium. Identification of the organism was made from culture using D1/D2 region of Large Sub Unit (28S rDNA) based molecular technique. Polymerase chain reaction amplified a band with a sequence that was $100 \%$ homologous with Lasiodiplodia theobromae. The organism was sensitive to amphotericin B and voriconazole, and demonstrated resistance to itraconazole and fluconazole. Therapeutic keratoplasty was performed followed by recurrence in graft controlled with topical voriconazole and intracameral amphotericin B. Graft failure was reported after three months.
\end{abstract}

\section{Introduction}

Lasiodiplodia theobromae is a plant pathogen with a very wide host range. It causes rotting and dieback in most species it infects. On rare occasions it has been found to cause fungal keratitis. Only 10 cases have been described in the international literature. ${ }^{1-5}$ In most of these cases, outcome was good. Therapeutic penetrating keratoplasty was necessary in 2 cases, and perforation occurred in 2 cases. We report the first case of infective keratitis caused by Lasiodiplodia theobromae from a tertiary-level eye care center in eastern India.

\section{Case Report}

A 32-year old female paddy-field worker presented with pain, redness and watering in the left eye of seven days duration following minor trauma with a woodstick 18 days prior to the onset of symptoms. She was using topical moxifloxacin, fortified tobramycin, natamycin and fluconazole before referral. At presentation, the best-corrected visual acuity in her left eye was perception of light with accurate projection of rays in all quadrants. Slit lamp examination revealed a corneal infiltrate measuring $9.8 \times 8.2 \mathrm{~mm}$ in size. Peripheral branching infiltrates with central raised plaque lesion was seen with hypopyon of $2.8 \mathrm{~mm}$ (Figure 1). An area of thinning was noted in the central portion of the infiltrate. No pigmentation or vascularization was noted. Right eye examination was within normal limits.

A standard microbiological workup was performed; $10 \%$ potassium hydroxide and Gram's staining showed refractile hyphae with asepted branching filaments (Figure 2). Topical treatment with $2 \%$ voriconazole and natamycin were started half hourly and atropine $1 \%$ eye drop was used three times a day. Systemic therapy consisted of oral ketoconazole $200 \mathrm{mg}$ twice daily. The patient was admitted for medication.

On medical therapy, there was an increase in the size of infiltrate with encroachment up to the limbus along with progression of central thinning requiring a therapeutic keratoplasty six days after initial presentation. The corneal button was also subjected to microbiological and histopathological examination.

After $72 \mathrm{~h}$ of incubation, large smooth black fluffy colonies with abundant aerial mycelium were grown on Saboraund's Dextrose Agar and Potato Dextrose Agar. The reverse side of the colonies was black pigmented. Lactophenol cotton blue mount from the colonies showed unicellular, hyaline subovoid thick walled mature conidia. The fungi was initially identified as a Lasiodiplodia sp. Species confirmation was carried out at the Xcelris Genomic Centre, Ahmedabad, India, by using D1/D2 region of Large Sub Unit (28SrDNA) based on polymerase chain reaction (PCR) technique. PCR amplified a band with a sequence that was $100 \%$ homologous with Lasiodiplodia theobromae, VTT C-04519 (GenBank Accession n. DQ377634.1) based on nucleotide homology and phylogenetic analysis.

Antifungal sensitivity testing was performed by broth microdilution method (CLSI document M27-A3). The organism was sensitive to amphotericin B and voriconazole with MIC values of $4 \mu \mathrm{g} / \mathrm{mL}$ and $1 \mu \mathrm{g} / \mathrm{mL}$, respectively. Resistance was demonstrated against itraconazole and fluconazole. Recurrence of infection in the graft was noted after three days with isolation of the same organism which was treated with topical voriconazole along with amphotericin B lavage of anterior chamber. This led to the reappearance of the infection and subsequently to graft failure after three months.
Correspondence: Suman Saha, Ocular Microbiology Services, Priyamvada Birla Aravind Eye Hospital, 10 Loudon Street, Kolkata 700017, West Bengal, India.

Fax: +91.33 .22894300$

E-mail: sumansaha13@gmail.com

Key words: keratitis, Lasiodiplodia sp.

Conflict of interests: the authors report no potential conflict of interests.

Received for publication: 5 November 2011. Accepted for publication: 11 April 2012.

This work is licensed under a Creative Commons Attribution NonCommercial 3.0 License (CC BYNC 3.0).

(C) Copyright S. Saha et al., 2012

Licensee PAGEPress, Italy

Microbiology Research 2012; 3:e19

doi:10.4081/mr.2012.e19

\section{Discussion}

This case draws our attention primarily because of the rarity of its occurrence and adds to our understanding of the disease process in the eye. Though the first case of Lasiodiplodia infection was reported by Patouillard et al. in 1892 in Ecuador and the first keratitis was reported by Rebell et al. ${ }^{1}$ way back in 1976 , among 4 cases only one required therapeutic keratoplasty. Until now, there have been more cases of keratitis $(n=2), 1,2$ than of endophthalmitis $(\mathrm{n}=3)^{3-5}$ Lasiodiplodia theobromae was responsible foro infection in only $5.12 \%$ of cases of total fungal keratitis in southern India.

This case involves a young immune competent individual with no identifiable risk factors other than minor trauma with a woodstick into the eye prior to the incidence. Identification of Lasiodiplodia theobromae was made in culture characterized by typical colony morphology and appearance of thick walled conidia. Confirmation of Lasiodiplodia theobromae was made by PCR-based DNA sequencing. This species was identified in keratitis (2 cases) as a common fungi in previous reports. ${ }^{1,2}$

Evaluation of sensitivity pattern for this organism showed in vitro activity of voriconazole and amphotericin B only. There are no sensitivity data available for Lasiodiplodia theobromae and this has an important bearing on the management of the case which showed lack of response to topical therapy with these antifungals. Similarly, failure of medical therapy in Lasiodiplodia keratitis was reported by Borderie et al. and Rebell et al. Though there ought to be differences in the severity of initial 


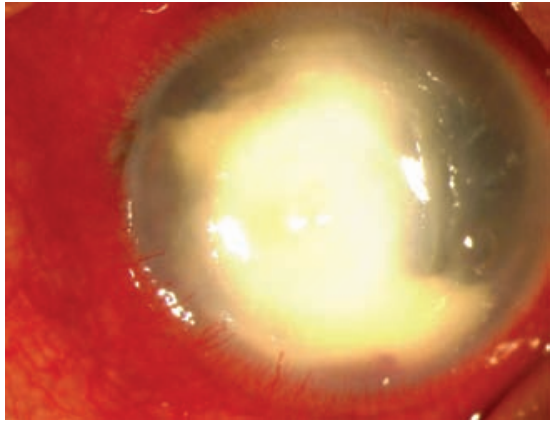

Figure 1. Clinical photo of Lasiodiplodia theobromae keratitis.

presentation amongst these cases, failure of medical therapy together with the sensitivity pattern of this isolate shows the inherent difficulties in treatment compared to systemic infections. In addition, the sensitivity data obtained in this case may reflect a pattern for the particular isolated strain only. Though recurrence of infection was controlled with topical voriconazole and amphotericin B therapy, it is difficult to comment upon the role of these two antifungals in Lasiodiplodia theobromae keratitis from a single case report. However, voriconazole has shown good intraocular penetration and in vitro activity against Lasiodiplodia theobromae. Amphotericin $B$ has been reported to be the most effective fungicidal treatment against $L$. theobromae. ${ }^{1}$ This combination therapy may become the mainstay therapy for the future as further evidence of its usefulness is provided and appropriate therapeutic principles become established.

\section{Conclusions}

Lasiodiplodia theobromae is a rare cause of fungal keratitis. Management of these cases is difficult often requiring surgical procedures, probably because of our lackof preparation in dealing with these organisms. Topical treatment with voriconazole may be considered in

\section{Electropherogram data file detail:}

The following Electropherogram files will be attached separately along with this report:

1. Sample_1-DF.ab1: Sequence obtained with forward primer

2. Sample_1-DR.ab1: Sequence obtained with reverse primer

Details of Sequences Generated:

Sample_1-DF

ATTGCCTTAGTAACGGCGAGTGAAGCGGCAACAGCTCAAATTGAAAGCTGGCCCTTITAGGGTCOGCATTG TAATITGTAGAGGATGATTCGGCGAGGGCTCCTGCCTAAGTCCCCTGGAACGGGGOGTCATAGAGGGTGAG AATCCCGTATGCGGTAGGTTGCCTTAGCCATGTGAATCTCCTTCGACGAGTCGAGTTGTTTGGGAATGCAGC TCTAAATG G GAGGTAAATIT CTTCTAAAGCTAAATAC CGGCCAGAGACCGATAGCGCACAAGTAGAGTGATCG AAAGATGAAAAGCACTTTGGAAAGAGAGTTAAAAAGTACGTGAAATTGTTGAAAGGGAAGCGCTTGCAACCA GACTTGTCCGCAGTTGCTCAGCCGGTCTCCTGACCGGOGTACTCTTCTGCGGCCAGGCCAGCATCAGTTCG GGCGGTCGGATAAAGGCCTCGGGAATGTGGCTOCTCTOGGGGAGTGTTATAGCCCGGGGTGCAATGCGGC CAGCCTGGACTGAGGAACTCGCTTCGGCAAGGATGCTGGCGTAATGGTTGTAAGCGGCOCGTCTTGAA

\section{Sample_1-DR}

CGAAGCGAGTTCCTCAGTCCAGGCTGGCCGCATTGCACCCCGGGCTATAACACTCCCCGAGAGGAGCCAC ATTCCCGAGGCCTITATCCGACCGCCCGAACTGATGCTGGCCTGGCCGCAGAAGAGTACGCCGGTCAGGA GACCGGCTGAGCAACTGCGGACAAGTCTGGTTGCAAGCGCTTCCCTICAACAATITCACGTACTITTAAC TCTCTTTCCAAAGTGCTTTCATCTTCGATCACTCTACTTGTGCGCTATCGGTCTCTGGCCGGTATTTAGCTT TAGAAGAAATTTACCTCCCATTTAGAGCTGCATT COCAAACAACTCGACTCGTCGAAGGAGATTCACATGGCT AAGGCAACCTACCGCATAOGGGATTCTCACCCTCTATGACGCCOCGTTCCAGGGGACTTAGGCAGGAGCCC TCGCCGAATCATCCTCTACAAATTACAATGCGGACCCTAAAAGGGCCAGCTITCAAATTTGAGCTGTTGCCGC TTCACTCGCCGTTACTAAGGCAATCCCTGTTGGTITCTITCCTCCGCTTATTGATATGCTTAAGTTCAG

\section{CONTIG REGION OF Sample-1}

CTGAACTTAAGCATATCAATAAGCGGAGGAAAAGAAACCAACAGGGATTGCCTTAGTAACGGCGAGTGAAGC GGCAACAGCTCAAATITGAAAG CTGG COCTITIAGGGTOCGCATTGTAATITGTAGAGGATGATTCGGCGAG GGCTCCTGCCTAAGTCCCCTGGAACGGGGCGTCATAGAGGGTGAGAATCCCGTATGCGGTAGGTTGCCTTA GCCATGTGAATCTCCTTCGACGAGTCGAGTTGTTTGGGAATGCAGCTCTAAATGGGAGGTAAATTCTTCTAA AGCTAAATACCGGCCAGAGACCGATAGCGCACAAGTAGAGTGATOGAAAGATGAAAAGCACTTTGGAAAGAG AGTTAAAAAGTACGTGAAATTGTTGAAAGGGAAGCGCTTGCAACCAGACTTGTCCGCAGTTGCTCAGCCGGT CTCCTGACCGGCGTACTCTTCTGCGGCCAGGOCAGCATCAGTTCGGGCGGTCGGATAAAGGCCTCGGGAA TGTGGCTCCTCTCGGGGAGTGTTATAGCCCGGGGTGCAATGCGGCCAGCCTGGACTGAGGAACTCGCTTC GGCAAGGATGCTGGCGTAATGGTTGTAAGCGGCCCGTCTTGAA

Figure 2. Chromatograme data with both primer and gel image of polymerase chain reaction amplicon.

non-responsive cases. Further reports are necessary to understand the disease and the organism in order to establish a treatment protocol.

\section{References}

1. Rebell G, Forster RK. Lasiodiplodia theobromae as a cause of keratomycoses. Sabouraudia 1976;14:155-70.

2. Thew MR, Todd B. Fungal keratitis in far north Queensland, Australia. Clin Experiment Ophthalmol 2008;36:721-4.

3. Slomovic AR, Forster RK, Gelender H. Lasiodiplodia theobromae panophthalmitis. Can J Ophthalmol 1985;20:225-8.

4. Donnio A, Desbois N, Boiron P, et al. Mycotic keratitis and endophthalmitis caused by unusual fungi: lasiodiplodia theobromae. J Fr Ophtalmol 2006;29:e4.

5. Borderie VM, Bourcier TM, Poirot JL, et al. Endophthalmitis after Lasiodiplodia theobromae corneal abscess. Graefes Arch Clin Exp Ophthalmol 1997;235:259-61. 